Relations industrielles

Industrial Relations

\title{
Coplanam Ltée. Index et résumés des sentences arbitrales de
} griefs, tome VII

\section{Rodrigue Blouin}

Volume 47, numéro 2, 1992

URI : https://id.erudit.org/iderudit/050779ar

DOI : https://doi.org/10.7202/050779ar

Aller au sommaire du numéro

\section{Éditeur(s)}

Département des relations industrielles de l'Université Laval

\section{ISSN}

0034-379X (imprimé)

1703-8138 (numérique)

Découvrir la revue

Citer ce compte rendu

Blouin, R. (1992). Compte rendu de [Coplanam Ltée. Index et résumés des sentences arbitrales de griefs, tome VII]. Relations industrielles / Industrial Relations, 47(2), 380-380. https://doi.org/10.7202/050779ar

Tous droits réservés (C Département des relations industrielles de l'Université Laval, 1992
Ce document est protégé par la loi sur le droit d'auteur. L’utilisation des services d'Érudit (y compris la reproduction) est assujettie à sa politique d'utilisation que vous pouvez consulter en ligne.

https://apropos.erudit.org/fr/usagers/politique-dutilisation/ 
helpful concluding essay. The essay ends by posing the key question of whether the hardships faced by the textile communities of the South are the growing pains of a dynamic global economy which will confer widespread benefits or whether the suffering of the southern textile workers is part of a global pattern of bipolarization with the rich getting richer and the poor getting poorer.

Irwin M. Marcus

Indiana University of Pennsylvania

Index et résumés des sentences arbitrales de griefs, tome VII, Montréal, Coplanam Itée, 1991, 755 p., ISBN 2-920391-04-6

Publiée pour la première fois en 1982, cette septième édition est demeurée fidèle à l'objectif initialement recherché, à savoir offrir un instrument de repérage des sentences arbitrales de griefs. Si, à l'époque, cet ouvrage comblait indéniablement un vide dans la documentation existante, if doit maintenant composer avec une forte concurrence notamment de la part de Soquij. En un tel contexte le service offert par Coplanam a certes encore son utilité mais il conviendrait de faire davantage.

Des trois catégories d'index qui constituent la première partie du recueil, le plus utile est indéniablement celui de repérage. Il se présente, tout comme d'ailleurs les index des arbitres et des présidents, sous forme d'une consolidation des index de 1970 à 1990. Pour connaître les résumés des sentences, il faut toujours manipuler l'ensemble des tomes. Les index renvoient par ailleurs aux numéros des sentences, lesquelles sont présentées par ordre numérique dans les sept recueils. Cette méthode oblige à feuilleter chaque volume pour localiser tel ou tel résumé. La consultation est loin d'être facilitée. La sentence arbitrale peut, quant à elle, être achetée du Bureau du commissairegénéral du travail ou de Coplanam. La seconde partie comprend d'abord des résumés de sentences arbitrales rendues en 1989 et en 1990. Les critères de sélection des sentences résumées ne sont pas publiés. Des listes des décisions 1989-1990 par ordre alphabétique de parties et par thèmes complètent cette seconde tranche de l'ouvrage.

Une telle manipulation de documents et les coûts impliqués pour obtenir les sentences risquent de décourager l'utilisateur et de l'inciter à joindre les rangs de ceux qui privilégient dorénavant les services informatisés ou ne serait-ce que les recueils sous formes mobiles. Le service offert par Coplanam n'est certes pas à négliger. Néanmoins il ne facilite plus autant la collecte de données qu'il y a quelques années. Pour rencontrer son objectif initial, cet éditeur doit dorénavant donner un sérieux coup de barre en repensant la présentation matérielle de sa documentation.

Rodrigue BLOUIN

Université Laval 